\title{
Evaluation of Cerebrovascular Reserve Capacity in Symptomatic and Asymptomatic Internal Carotid Stenosis With Transcranial Doppler
}

\author{
I. DOUVAS ${ }^{\mathbf{1}^{*}}$, D. MORIS ${ }^{\mathbf{*}}$, G. KARAOLANIS ${ }^{\mathbf{1}}$, C. BAKOYIANNIS $^{\mathbf{1}}$, S. GEORGOPOULOS $^{\mathbf{1}}$ \\ *These authors contributed equally. \\ ${ }^{1}$ First Department of Surgery, Vascular Surgery Unit, Laikon General Hospital, School of Medicine, \\ Athens, Greece
}

Received January 25, 2016

Accepted April 29, 2016

On-line August 19, 2016

\begin{abstract}
Summary
Cerebrovascular reserve capacity (CVRC) is a hemodynamic parameter indicating the brain's capacity to overcome ischemia. Transcranial Doppler (TCD) is a useful device to measure CVRC, with high availability and low cost. The aim of the study is to investigate asymptomatic patients with affected CVRC, who could benefit from CEA. One hundred and forty five consecutive patients (60 symptomatic and 65 asymptomatic), with internal carotid artery (ICA) stenosis $>70 \%$ and 20 healthy individuals without internal carotid stenosis underwent TCD-inhalation $\mathrm{CO}_{2}$ tests in order to measure the CVRC in both hemispheres of each patient. CVRC between asymptomatic and symptomatic patients were significantly different in the $95 \%$ confidence interval (CI) as well as the mean CVRC value in contralateral carotid artery. The correlation between CVRC in the carotid artery with stenosis and the existence of symptoms is significant at the 0.01 level. Additionally, symptoms and CVRC of the contralateral carotid artery are also significant at the 0.05 level and CVRC values in asymptomatic patients and the control group at the 0.01 level. None of the covariant factors, except the age, are significantly correlated with CRVC. CVRC could be an early mark-index to evaluate the risk of stroke in this group of patients and to design their therapeutic approach.
\end{abstract}

\section{Key words}

Cerebrovascular reserve capacity - Transcranial Doppler • Internal carotid stenosis • Carotid endarterectomy

\section{Corresponding author}

D. N. Moris, First Department of Surgery, Vascular Surgery Unit, Laikon General Hospital, School of Medicine,
Anastasiou Gennadiou 56, 11474, Athens, Greece. E-mail: dimmoris@yahoo.com

\section{Introduction}

Large prospective, randomized controlled trials have proved the benefit of carotid endarterectomy (CEA) especially in symptomatic and asymptomatic patients with severe carotid stenosis (70-99 \%) (ECST trial 1998, Ferguson et al. 1999). In these studies, a number of subgroups of patients had been identified, in which CEA could be beneficial in preventing stroke.

Beyond the criterion of the degree of ICA stenosis, several factors may affect the risk of stroke, such as gender, age, dyslipidemia, smoking, diabetes, hypertension and the type of the initial ischemic attack (TIA, symptoms $>24 \mathrm{~h}$, the presence of an infarct) (Shaikh et al. 2010). This may be explained by changes in cerebral hemodynamic parameters. Impaired cerebral perfusion is associated with higher incidence of TIA or stroke (Zachrisson et al. 2012).

The cerebrovascular reserve capacity (CVRC) reflects the hemodynamic status of the cerebral circulation and could be a useful tool to identify which patients are at higher risk of stroke. There are many methods evaluating CVRC such as transcranial Doppler (TCD), magnetic resonance imaging (MRI), positron emission tomography (PET) and single-photon emission computed tomography (SPECT) (Tsivgoulis and Alexandrov 2008). When compared with other methods, TCD is thought to be less expensive method with a higher 
availability (Tsivgoulis and Alexandrov 2008).

The aim of the present study is to investigate asymptomatic patients with affected CVRC who could be benefited from CEA.

\section{Materials and Methods}

We prospectively studied 125 consecutive patients whose CVRC was measured with TCD. All patients had unilateral ICA stenosis $>70 \%$, that was evaluated preoperatively with duplex scanning by the same investigator. A careful neurological and cardiologic examination including electrocardiogram, transthoracic echocardiography and brain computed tomography (CT) scan was also performed in all the participants of the study. Also, complete blood examination and clinical history with particular attention to the major vascular risk factors (hypertension, diabetes, smoking and hyperlipidemia) was obtained from each patient.

Exclusion criteria were (Silvestrini et al. 1996):

a) history of thrombophilia, idiopathic or hereditary,

b) bilateral severe carotid stenosis, c) heart failure, atrial fibrillation and valve disease, d) ascending aorta aneurysms, e) brain pathology (tumors, arterio-vein communications, aneurysms, mental disturbances), f) obstructive pulmonary disease, g) hematocrit $<35 \%$.

Sixty-five patients were asymptomatic (group A), while 60 patients were symptomatic (group B). As symptomatic were defined the patients who had suffered from carotid distribution transient ischemic attack (TIA) or a non-disabling stroke in the preceding 6 months. Patients with silent cerebral infarct in CT-scan were also considered symptomatic. Twenty healthy individuals were used as a control group (group C) and they were also evaluated with detailed neurological examination and duplex scanning by the same investigator. We considered as "healthy" the individuals who did not suffer from cerebrovascular or cardiovascular diseases and they did not present with abnormal findings on the duplex and CT scanning examination.

Symptomatic patients were further classified according the duration of their symptoms (TIA, symptoms lasting $>24 \mathrm{~h}$, stroke), cerebral CT-scan findings (presence or absence of an ischemic infarct) and the degree of carotid stenosis (70-79\%, 80-89\%, 90-99\%).

Each patient was examined early in the morning on the day before CEA. The patients had been instructed to avoid coffee, alcohol, refreshments and smoking for the last $12 \mathrm{~h}$ (Silvestrini et al. 1996, Nemoto et al. 2004). Before TCD measurements, arterial blood gases and blood pressure were measured. The upper limit for systolic arterial pressure was $130 \mathrm{~mm} \mathrm{Hg}$, otherwise it was regulated.

Measurements were performed at rest and after an administration of a mixture of $95 \% \mathrm{O}_{2}$ and $5 \% \mathrm{CO}_{2}$, from the temporal window, bilaterally. Patients breathed through the ventilation mask until middle cerebral artery (MCA) velocity became stable. Then, recording was continued for $30 \mathrm{~s}$. CVRC was estimated by the following equation:

$$
\text { CVRC }=\frac{\text { MVFCO2 }- \text { MVFrest }}{\text { MVFrest }} \times 100
$$

where MVFCO2 is middle velocity flow, in the middle cerebral artery during the mixture inhalation and MVFrest is middle velocity flow, in the middle cerebral artery during rest.

\section{Statistical analysis}

Statistical analysis was performed using the Statistical Package for Social Sciences (SPSS version 17.0; SPSS, Chicago, IL) software. Statistical significance was set at $\mathrm{p}<0.05$. Values are expressed as mean percentage signal change \pm standard deviation (SD). Two-tailed test was performed for establishing statistically significant or no correlation. Test for normality (Kolmogorov-Smirnov) was used to confirm the normal distribution of the data. A univariate analysis of covariance (ANCOVA) was performed to look at the effect on CVRC (as assessed by TCD) whilst controlling for age, hypertension, type of ischemic attack (TIA, symptoms $>24 \mathrm{~h}$, the presence of an infarct), hyperlipidemia, diabetes, gender and smoking.

\section{Results}

A total of 145 patients were deemed appropriate for inclusion in this study, 65 were asymptomatic (mean age $70.45 \pm 4.66$ ), 60 were symptomatic (mean age $74.5 \pm 3.83$ ) and 20 were "healthy" individuals (mean age $65.85 \pm 3.31$ ). The mean CVRC value for the symptomatic patients was $14.37 \pm 5.66 \%$ and in asymptomatic was $19.18 \pm 5.72 \%$. These values were significantly different $(p=0.01)$. The mean CVRC value in contralateral carotid artery for the symptomatic patients was $21.96 \pm 2.44 \%$ and in asymptomatic was $23.38 \pm 3.53 \%$. These values 
were also significantly different $(\mathrm{p}=0.05)$. The mean CVRC value for the control group was $24.87 \pm 4.60 \%$ (Fig. 1). The lowest CRVC value in this group was $20.27 \%$. Values below $20.27 \%$ were considered as abnormal or affected. Interestingly, there was a statistically significant correlation between the CVRC values in asymptomatic patients and the control group $(\mathrm{p}<0.01)$.

Regarding both groups, the difference between the percentage of patients with affected CVRC in all categories of stenosis was significant. More specifically, the percentage of patients with affected CVRC in the first subcategory (70-79\% stenosis), was $32.7 \%$, whereas in

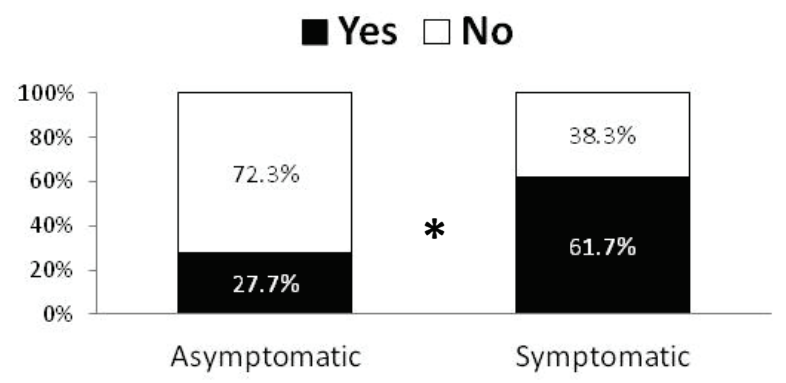

Fig. 1. Incidence of affected CVRC in symptomatic and asymptomatic patients. $* \mathrm{p}<0.05$ significantly different.

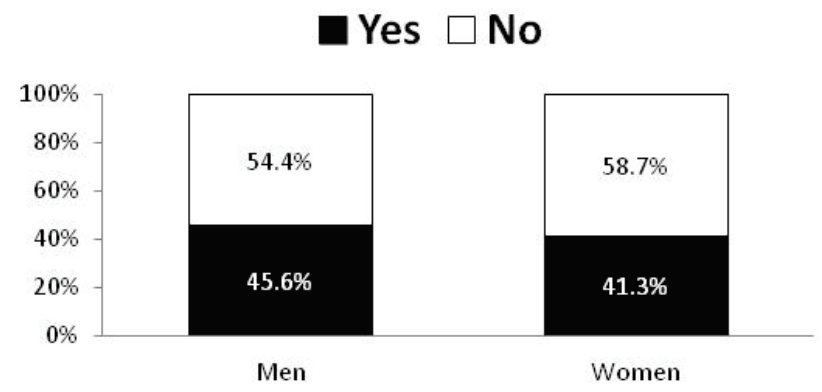

Fig. 3. The influence of gender on the incidence of affected CVRC.

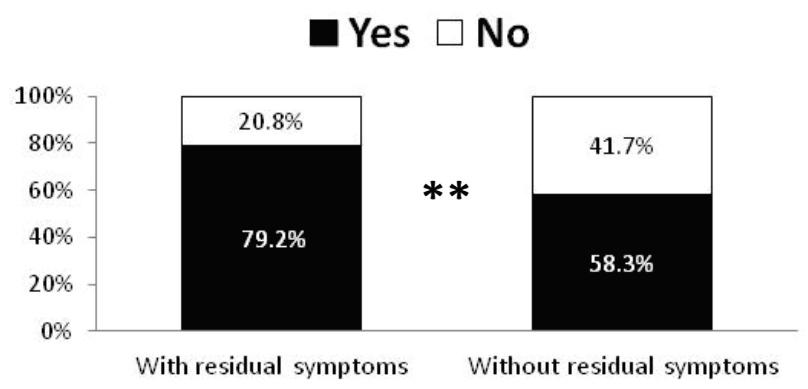

Fig. 5. The relation of residual symptoms to the incidence of affected CVRC. $* * \mathrm{p}<0.01$ significantly different. the $80-89 \%$ category was $45.5 \%$ and in the $90-99 \%$ category was $59.4 \%(p<0.05)$ (Fig. 2$)$. There was no difference found between the percentage of men and women with affected CRVC (45.6\% vs. $41.3 \%$ respectively) (Fig. 3).

In group $\mathrm{B}$, among patients with symptoms $>24 \mathrm{~h}$ and TIA, the affected CVRC was $72.22 \%$ and $45.8 \%$ respectively $(\mathrm{p}<0.01)$ (Fig. 4$)$, whereas within the sub-group with neurological symptoms $>24 \mathrm{~h}, 79.16 \%$ of patients with residual symptoms presented with affected CVRC, whereas in patients without residual symptoms only $58.33 \%$ of them presented with affected CRVC $(\mathrm{p}<0.05)$ (Fig. 5) (Table 1).

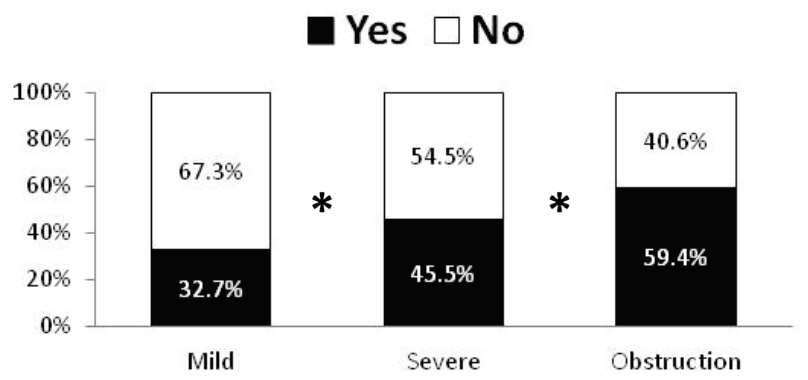

Fig. 2. Incidence of affected CVRC in relation to the severity of the symptoms. $*$ p $<0.05$ significantly different.

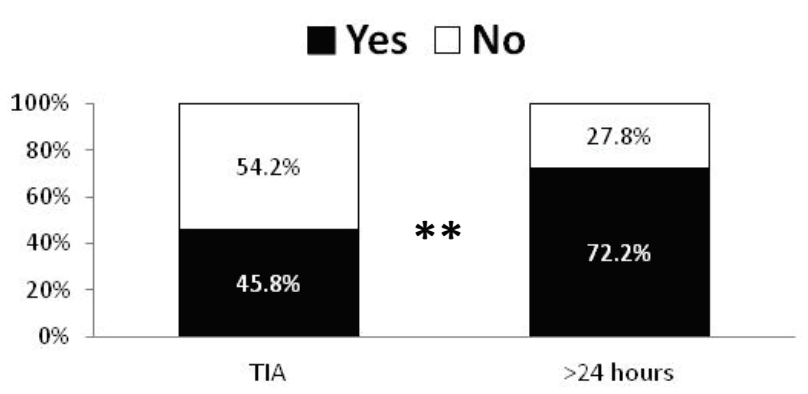

Fig. 4. Incidence of affected CVRC in patients with transient ischemic attack (TIA) or ischemic attack lasting longer than $24 \mathrm{~h}$. $* * \mathrm{p}<0.01$ significantly different.

As far as the type of the cerebrovascular disease is concerned, our findings indicated no significant correlation between the CVRC values and the type of ischemic attack. Specifically, the affected CRVC values in the subgroup of symptomatic patients with TIA did not significantly differ from the relevant values in the subgroup of patients with symptoms $>24 \mathrm{~h}$ (with or without residual symptoms) $(\mathrm{p}=0.056)$ (Table 2$)$. The presence of an infarct and its correlation with the affected CRVC values was also not significant $(p=0.340)$ 
(Table 3). None of the covariant factors such as gender $(p=0.819)$, dyslipidemia $(p=0.440)$, smoking $(p=0.368)$, diabetes $(p=0.351)$ and hypertension $(p=0.779)$ were significantly correlated with $\mathrm{CRVC}$ as well as after a univariate analysis of covariance (ANCOVA).

As far as age is concerned, it was significantly correlated with CRVC at the 0.01 level in the two-tailed test but this significance cannot be reconfirmed after ANCOVA $(p=0.033)$. Lastly, there is no respectable ascendancy recorded between the co-existent diseases, diabetes, hyperlipidemia, hypertension and smoking and the affected CVRC.

Table 1. Data of the patients with symptoms $>24 \mathrm{~h}$.

\begin{tabular}{|c|c|c|c|c|c|c|c|c|c|c|c|c|}
\hline Gender & Age & MVFrest & MVFCO2 & CRC & Category & Diabetes & Hypertension & Smoking & Dyslipidemia & Stenosis & Infarct & $\begin{array}{c}\text { Affected } \\
\text { CRVC }\end{array}$ \\
\hline M & 79 & $51-53$ & $57-64$ & $11.7-20.7$ & RS & Y & Y & Y & $\mathrm{N}$ & M & Y & Y \\
\hline M & 80 & $40-43$ & $45-52$ & $12.5-20.93$ & RS & $\mathrm{Y}$ & Y & $\mathrm{N}$ & $\mathrm{Y}$ & M & $\mathrm{Y}$ & $\mathrm{Y}$ \\
\hline M & 77 & $41-44$ & $45-53$ & $9.7-20.45$ & RS & $\mathrm{Y}$ & Y & Y & $\mathrm{Y}$ & M & Y & $\mathrm{Y}$ \\
\hline M & 69 & $51-56$ & $56-67$ & $9.8-19.29$ & RS & $\mathrm{Y}$ & $\mathrm{Y}$ & $\mathrm{Y}$ & $\mathrm{N}$ & M & $\mathrm{Y}$ & $\mathrm{Y}$ \\
\hline M & 72 & $54-56$ & $59-65$ & $9.25-17.8$ & RS & $\mathrm{Y}$ & $\mathrm{Y}$ & $\mathrm{N}$ & $\mathrm{Y}$ & M & $\mathrm{Y}$ & $\mathrm{Y}$ \\
\hline F & 78 & $51-52$ & $56-63$ & $9.6-21.1$ & RS & $\mathrm{N}$ & $\mathrm{N}$ & Y & $\mathrm{N}$ & S & Y & $\mathrm{Y}$ \\
\hline $\mathrm{F}$ & 80 & $47-48$ & $51-60$ & $9.3-25.58$ & RS & Y & $\mathrm{N}$ & Y & Y & S & $\mathrm{Y}$ & Y \\
\hline F & 78 & $41-42$ & $45-52$ & $9.75-23.8$ & RS & $\mathrm{N}$ & $\mathrm{N}$ & $\mathrm{N}$ & $\mathrm{Y}$ & S & $\mathrm{Y}$ & $\mathrm{Y}$ \\
\hline $\mathrm{M}$ & 76 & $43-42$ & $51-50$ & $18.6-19.04$ & RS & $\mathrm{N}$ & $\mathrm{Y}$ & Y & $\mathrm{N}$ & M & $\mathrm{Y}$ & $\mathrm{N}$ \\
\hline M & 74 & $58-56$ & 64-68 & $10.3-21.4$ & RS & Y & $\mathrm{N}$ & $\mathrm{Y}$ & $\mathrm{N}$ & S & $\mathrm{Y}$ & Y \\
\hline M & 77 & $46-47$ & $51-56$ & $10.8-21.7$ & RS & $\mathrm{Y}$ & $\mathrm{N}$ & $\mathrm{Y}$ & $\mathrm{N}$ & S & $\mathrm{Y}$ & $\mathrm{Y}$ \\
\hline M & 74 & $38-41$ & $42-51$ & $10.52-24.39$ & RS & $\mathrm{N}$ & Y & $\mathrm{N}$ & $\mathrm{N}$ & S & $\mathrm{Y}$ & $\mathrm{Y}$ \\
\hline $\mathrm{F}$ & 75 & $40-44$ & $45-53$ & $9-20.4$ & RS & $\mathrm{N}$ & Y & $\mathrm{Y}$ & $\mathrm{Y}$ & S & $\mathrm{Y}$ & Y \\
\hline M & 76 & $43-42$ & $51-50$ & $18.6-19.04$ & RS & $\mathrm{Y}$ & $\mathrm{N}$ & $\mathrm{N}$ & $\mathrm{N}$ & M & $\mathrm{Y}$ & $\mathrm{N}$ \\
\hline M & 77 & $49-50$ & $54-61$ & $10.2-22$ & RS & $\mathrm{Y}$ & $\mathrm{Y}$ & Y & $\mathrm{N}$ & $\mathrm{O}$ & $\mathrm{Y}$ & Y \\
\hline F & 78 & $43-47$ & $47-57$ & $8.51-21.27$ & RS & $\mathrm{Y}$ & $\mathrm{N}$ & $\mathrm{N}$ & $\mathrm{N}$ & $\mathrm{O}$ & $\mathrm{Y}$ & $\mathrm{Y}$ \\
\hline F & 79 & $39-40$ & $43-48$ & $10.2-20$ & RS & $\mathrm{N}$ & Y & Y & $\mathrm{Y}$ & o & Y & $\mathrm{Y}$ \\
\hline M & 71 & $46-47$ & $50-57$ & $8.7-21.2$ & RS & $\mathrm{N}$ & Y & $\mathrm{N}$ & Y & $\mathrm{O}$ & Y & $\mathrm{Y}$ \\
\hline M & 72 & $54-55$ & $66-67$ & $22.2-21.8$ & RS & $\mathrm{Y}$ & Y & $\mathrm{N}$ & $\mathrm{N}$ & M & $\mathrm{Y}$ & $\mathrm{N}$ \\
\hline M & 75 & $51-56$ & $56-67$ & $9.8-19.29$ & RS & $\mathrm{N}$ & $\mathrm{Y}$ & Y & $\mathrm{N}$ & o & Y & $\mathrm{Y}$ \\
\hline M & 71 & $43-46$ & $54-57$ & $25.58-23.9$ & RS & $\mathrm{Y}$ & $\mathrm{Y}$ & Y & Y & $\mathrm{S}$ & $\mathrm{Y}$ & $\mathrm{N}$ \\
\hline F & 70 & $41-45$ & $50-56$ & 21.9-24.44 & RS & $\mathrm{Y}$ & $\mathrm{N}$ & $\mathrm{N}$ & $\mathrm{Y}$ & S & $\mathrm{Y}$ & $\mathrm{N}$ \\
\hline $\mathrm{M}$ & 74 & $55-57$ & 61-68 & $10.9-19.2$ & RS & $\mathrm{N}$ & $\mathrm{Y}$ & Y & $\mathrm{N}$ & $\mathrm{O}$ & $\mathrm{Y}$ & Y \\
\hline M & 69 & $41-43$ & $47-54$ & $14.6-25.58$ & WRS & $\mathrm{N}$ & $\mathrm{Y}$ & Y & $\mathrm{N}$ & M & Y & $\mathrm{Y}$ \\
\hline M & 79 & $43-48$ & $54-60$ & $25.58-25$ & WRS & $\mathrm{Y}$ & $\mathrm{Y}$ & $\mathrm{N}$ & $\mathrm{Y}$ & M & $\mathrm{Y}$ & $\mathrm{N}$ \\
\hline M & 75 & $41-44$ & $46-53$ & $12.1-20.45$ & WRS & $\mathrm{Y}$ & $\mathrm{Y}$ & Y & Y & M & $\mathrm{Y}$ & Y \\
\hline M & 73 & $45-49$ & $56-60$ & $24.44-22.44$ & WRS & $\mathrm{N}$ & Y & $\mathrm{N}$ & Y & M & Y & $\mathrm{N}$ \\
\hline M & 76 & $48-49$ & $60-62$ & $25-26.5$ & WRS & $\mathrm{Y}$ & $\mathrm{N}$ & $\mathrm{N}$ & $\mathrm{N}$ & S & $\mathrm{Y}$ & $\mathrm{N}$ \\
\hline M & 77 & $44-47$ & $50-59$ & $13.63-25.53$ & WRS & $\mathrm{N}$ & $\mathrm{N}$ & Y & Y & $\mathrm{S}$ & $\mathrm{Y}$ & Y \\
\hline M & 78 & $48-53$ & $55-63$ & $10.4-18.86$ & WRS & $\mathrm{N}$ & $\mathrm{Y}$ & Y & $\mathrm{N}$ & $\mathrm{S}$ & $\mathrm{Y}$ & Y \\
\hline F & 70 & $51-53$ & $61-65$ & $19.6-22.6$ & WRS & $\mathrm{N}$ & $\mathrm{Y}$ & $\mathrm{N}$ & $\mathrm{Y}$ & S & Y & $\mathrm{N}$ \\
\hline $\mathrm{M}$ & 71 & $41-42$ & $45-51$ & $9.7-17.6$ & WRS & $\mathrm{N}$ & $\mathrm{N}$ & $\mathrm{N}$ & Y & $\mathrm{S}$ & Y & Y \\
\hline $\mathrm{M}$ & 68 & $49-50$ & $54-61$ & $10.2-22$ & WRS & $\mathrm{Y}$ & $\mathrm{N}$ & $\mathrm{N}$ & $\mathrm{N}$ & $\mathrm{O}$ & $\mathrm{Y}$ & Y \\
\hline $\mathrm{F}$ & 72 & $40-44$ & $48-59$ & $20-20.4$ & WRS & $\mathrm{Y}$ & Y & $\mathrm{N}$ & $\mathrm{N}$ & o & Y & $\mathrm{N}$ \\
\hline $\mathrm{F}$ & 66 & $47-48$ & $54-60$ & $14.89-25$ & WRS & $\mathrm{N}$ & $\mathrm{N}$ & Y & $\mathrm{Y}$ & o & Y & Y \\
\hline
\end{tabular}

$\mathrm{Y}$ - yes, $\mathrm{N}$ - no, $\mathrm{M}$ - mild, S - severe, O - occlusion, RS - residual symptoms, WRS - without residual symptoms, MVFCO2 - middle velocity flow, MVFrest - middle velocity flow, CRVC - cerebrovascular reserve capacity, M - male, F- female. 
Table 2. Data of the patients with TIA.

\begin{tabular}{|c|c|c|c|c|c|c|c|c|c|c|c|c|}
\hline Gender & Age & MVFrest & MVFCO2 & CRC & Category & Diabetes & Hypertension & Smoking & Dyslipidemia & Stenosis & Infarct & $\begin{array}{c}\text { Affected } \\
\text { CRVC }\end{array}$ \\
\hline M & 75 & $41-42$ & $50-50$ & 21.9-19.05 & TIA & $\mathrm{N}$ & $\mathrm{Y}$ & $\mathrm{N}$ & $\mathrm{N}$ & M & Y & $\mathrm{N}$ \\
\hline $\mathrm{F}$ & 71 & $51-52$ & $56-63$ & $9.8-21.1$ & TIA & $\mathrm{Y}$ & $\mathrm{N}$ & $\mathrm{Y}$ & $\mathrm{N}$ & M & Y & Y \\
\hline M & 70 & $53-55$ & $57-67$ & $7.5-21.8$ & TIA & $\mathrm{N}$ & $\mathrm{N}$ & $\mathrm{Y}$ & $\mathrm{Y}$ & M & & Y \\
\hline M & 69 & $43-42$ & $52-52$ & $21.4-23.8$ & TIA & $\mathrm{N}$ & $\mathrm{Y}$ & $\mathrm{N}$ & $\mathrm{N}$ & M & & $\mathrm{N}$ \\
\hline M & 70 & $39-40$ & $43-49$ & $10.2-22.5$ & TIA & $\mathrm{Y}$ & $\mathrm{N}$ & $\mathrm{N}$ & $\mathrm{Y}$ & M & & Y \\
\hline M & 70 & $52-53$ & $62-61$ & $19.2-20.7$ & TIA & $\mathrm{N}$ & $\mathrm{Y}$ & $\mathrm{N}$ & $\mathrm{N}$ & M & & $\mathrm{N}$ \\
\hline M & 68 & $54-55$ & $59-65$ & $9.2-18.18$ & TIA & Y & Y & $\mathrm{Y}$ & $\mathrm{N}$ & $\mathrm{S}$ & $\mathrm{Y}$ & $\mathrm{Y}$ \\
\hline $\mathrm{F}$ & 69 & $46-47$ & $50-57$ & $8.7-21.2$ & TIA & $\mathrm{N}$ & Y & $\mathrm{Y}$ & $\mathrm{Y}$ & $\mathrm{S}$ & & $\mathrm{N}$ \\
\hline M & 75 & $48-49$ & $60-62$ & $25-26.5$ & TIA & $\mathrm{N}$ & $\mathrm{Y}$ & $\mathrm{N}$ & $\mathrm{N}$ & M & & $\mathrm{Y}$ \\
\hline M & 76 & $43-43$ & $47-54$ & $9.3-25.58$ & TIA & $\mathrm{N}$ & $\mathrm{N}$ & $\mathrm{Y}$ & $\mathrm{Y}$ & S & & $\mathrm{N}$ \\
\hline $\mathrm{F}$ & 77 & $47-48$ & $51-60$ & $8.51-25$ & TIA & $\mathrm{Y}$ & $\mathrm{Y}$ & $\mathrm{Y}$ & $\mathrm{N}$ & $\mathrm{S}$ & & $\mathrm{N}$ \\
\hline M & 80 & $41-42$ & $45-52$ & $9.75-23.8$ & TIA & $\mathrm{Y}$ & $\mathrm{Y}$ & $\mathrm{N}$ & Y & $\mathrm{O}$ & & $\mathrm{N}$ \\
\hline M & 79 & $43-44$ & $51-52$ & $18.6-18.1$ & TIA & $\mathrm{N}$ & $\mathrm{Y}$ & $\mathrm{Y}$ & $\mathrm{N}$ & M & & $\mathrm{Y}$ \\
\hline M & 77 & $49-50$ & $53-60$ & $7.5-20$ & TIA & Y & Y & $\mathrm{N}$ & $\mathrm{Y}$ & $\mathrm{O}$ & & $\mathrm{Y}$ \\
\hline F & 80 & $38-39$ & $41-47$ & $7.8-20.5$ & TIA & $\mathrm{N}$ & $\mathrm{Y}$ & $\mathrm{N}$ & $\mathrm{N}$ & $\mathrm{O}$ & & Y \\
\hline $\mathrm{F}$ & 73 & $54-55$ & $66-67$ & $22.2-21.8$ & TIA & $\mathrm{Y}$ & $\mathrm{N}$ & $\mathrm{N}$ & $\mathrm{N}$ & M & & $\mathrm{N}$ \\
\hline $\mathrm{F}$ & 74 & $43-42$ & $51-50$ & 18.6-19.04 & TIA & $\mathrm{N}$ & $\mathrm{Y}$ & $\mathrm{N}$ & $\mathrm{N}$ & M & & $\mathrm{N}$ \\
\hline F & 75 & $39-42$ & $47-51$ & $19.04-17.64$ & TIA & $\mathrm{N}$ & Y & Y & Y & $\mathrm{S}$ & Y & $\mathrm{N}$ \\
\hline M & 77 & $41-42$ & $47-52$ & $14.63-23.8$ & TIA & $\mathrm{Y}$ & $\mathrm{N}$ & $\mathrm{N}$ & $\mathrm{N}$ & $\mathrm{O}$ & & $\mathrm{Y}$ \\
\hline M & 79 & $40-44$ & $48-53$ & $20-20.4$ & TIA & $\mathrm{Y}$ & $\mathrm{N}$ & $\mathrm{Y}$ & $\mathrm{N}$ & $\mathrm{S}$ & & $\mathrm{N}$ \\
\hline $\mathrm{F}$ & 80 & $40-45$ & $48-54$ & $20-20.5$ & TIA & $\mathrm{N}$ & $\mathrm{Y}$ & $\mathrm{Y}$ & $\mathrm{N}$ & $\mathrm{S}$ & & $\mathrm{N}$ \\
\hline $\mathrm{F}$ & 71 & $40-43$ & $49-52$ & $22.5-20.9$ & TIA & $\mathrm{Y}$ & $\mathrm{Y}$ & $\mathrm{Y}$ & $\mathrm{N}$ & $\mathrm{O}$ & $\mathrm{Y}$ & $\mathrm{N}$ \\
\hline M & 81 & $43-42$ & $52-52$ & $21.4-23.8$ & TIA & $\mathrm{Y}$ & $\mathrm{Y}$ & $\mathrm{N}$ & $\mathrm{Y}$ & $\mathrm{O}$ & & $\mathrm{N}$ \\
\hline $\mathrm{F}$ & 75 & $51-55$ & $63-68$ & $25.49-23.63$ & TIA & $\mathrm{N}$ & Y & $\mathrm{N}$ & $\mathrm{N}$ & $\mathrm{O}$ & & $\mathrm{N}$ \\
\hline
\end{tabular}

$\mathrm{Y}$ - yes, $\mathrm{N}$ - no, $\mathrm{M}$ - mild, S - severe, O - occlusion, TIA - transient ischemic attack, MVFCO2 - middle velocity flow, MVFrest - middle velocity flow, CRVC - cerebrovascular reserve capacity, $M-$ male, $F-$ female.

\section{Discussion}

Many multicenter studies (ECST trial 1998) (Ferguson et al. 1999) in patients with carotid stenosis have shown that in asymptomatic patients, the risk/benefit ratio of CEA is marginal. Our aim was to identify these specific subgroups of asymptomatic patients with affected CRVC, in which CEA could be beneficial. CRVC values below $20.27 \%$ were considered as affected.

The brain, in order to compensate the stenosis of intracranial and extracranial arteries, promotes the development or the utility of collateral circulation. If the collateral circulation could not maintain adequate blood flow, two other mechanisms emerge: the increase of oxygen extraction (Derdeyn et al. 1999, Donahue et al. 2014) and the vasodilation of cerebral arteries. Cerebrovascular reserve capacity is based on the vasodilation from the increase in the concentration of
$\mathrm{CO}_{2}$. Brain hemisphere without satisfactory collateral circulation, due to low blood flow, the arterioles are in maximum vasodilation, so that the challenge of further vasodilation may sustain little response and the price of CRVC that is measured should be low (Derdeyn et al. 1999, Donahue et al. 2014).

One interesting observation that arises is the fact that affected CVRC could not be found in the hemisphere with non-hemodynamically significant carotid stenosis $<30 \%$. Orosz et al. (2002) measured the "healthy" hemisphere and came up with similar findings. A possible explanation could be the fact that CVRC is liable to changes in hemodynamic stability that is not the case in these studies since the stenosis was below $30 \%$. A significant observation is noted between asymptomatic and symptomatic patients, where the difference regarding with affected CVRC is statistically significant.

The mean CRVC value for symptomatic patients was $14.37 \pm 5.66 \%$ whereas in asymptomatic was 
$19.18 \pm 5.72 \%$. These values were significantly different as well as the difference of mean CRVC values in contralateral carotid artery between symptomatic
$(21.96 \pm 2.44 \%)$ and in asymptomatic patients $(23.38 \pm 3.53 \%)$.

Table 3. Data of the patients with infarct.

\begin{tabular}{|c|c|c|c|c|c|c|c|c|c|c|c|c|}
\hline Gender & Age & MVFrest & MVFCO2 & CRC & Category & Diabetes & Hypertension & Smoking & Dyslipidemia & Stenosis & Infarct & $\begin{array}{c}\text { Affected } \\
\text { CRVC }\end{array}$ \\
\hline M & 73 & $52-54$ & $57-67$ & $9.6-24$ & RS & $\mathrm{Y}$ & $\mathrm{N}$ & $\mathrm{N}$ & $\mathrm{Y}$ & M & $\mathrm{Y}$ & $\mathrm{Y}$ \\
\hline M & 79 & $51-53$ & $57-64$ & $11.7-20.7$ & RS & $\mathrm{Y}$ & $\mathrm{Y}$ & $\mathrm{Y}$ & $\mathrm{Y}$ & M & $\mathrm{Y}$ & $\mathrm{Y}$ \\
\hline M & 80 & $40-43$ & $45-52$ & $12.5-20.93$ & RS & $\mathrm{N}$ & $\mathrm{Y}$ & $\mathrm{N}$ & $\mathrm{N}$ & M & $\mathrm{Y}$ & $\mathrm{Y}$ \\
\hline M & 77 & $41-44$ & $45-53$ & $9.7-20.45$ & RS & $\mathrm{N}$ & $\mathrm{Y}$ & $\mathrm{Y}$ & $\mathrm{N}$ & M & $\mathrm{Y}$ & $\mathrm{Y}$ \\
\hline M & 69 & $51-56$ & $56-67$ & $9.8-19.29$ & RS & $\mathrm{N}$ & $\mathrm{Y}$ & $\mathrm{N}$ & $\mathrm{Y}$ & M & $\mathrm{Y}$ & $\mathrm{Y}$ \\
\hline M & 72 & $54-56$ & $59-65$ & $9.25-17.8$ & RS & $\mathrm{N}$ & YN & $\mathrm{Y}$ & $\mathrm{N}$ & M & $\mathrm{Y}$ & $\mathrm{Y}$ \\
\hline $\mathrm{F}$ & 78 & $51-52$ & $56-63$ & $9.6-21.1$ & RS & $\mathrm{Y}$ & $\mathrm{N}$ & $\mathrm{N}$ & $\mathrm{Y}$ & $\mathrm{S}$ & $\mathrm{Y}$ & $\mathrm{Y}$ \\
\hline $\mathrm{F}$ & 80 & $47-48$ & $51-60$ & $9.3-25.58$ & RS & $\mathrm{N}$ & $\mathrm{N}$ & $\mathrm{N}$ & $\mathrm{N}$ & S & $\mathrm{Y}$ & $\mathrm{Y}$ \\
\hline F & 78 & $41-42$ & $45-52$ & $9.75-23.8$ & RS & $\mathrm{Y}$ & $\mathrm{N}$ & $\mathrm{Y}$ & $\mathrm{N}$ & S & $\mathrm{Y}$ & $\mathrm{Y}$ \\
\hline M & 76 & $43-42$ & $51-50$ & $18.6-19.04$ & RS & $\mathrm{N}$ & $\mathrm{Y}$ & $\mathrm{N}$ & $\mathrm{Y}$ & M & $\mathrm{Y}$ & $\mathrm{N}$ \\
\hline M & 74 & $58-56$ & $64-68$ & $10.3-21.4$ & RS & $\mathrm{Y}$ & $\mathrm{N}$ & $\mathrm{N}$ & $\mathrm{Y}$ & S & $\mathrm{Y}$ & $\mathrm{Y}$ \\
\hline M & 77 & $46-47$ & $51-56$ & $10.8-21.7$ & RS & $\mathrm{Y}$ & $\mathrm{N}$ & $\mathrm{N}$ & $\mathrm{Y}$ & S & $\mathrm{Y}$ & $\mathrm{Y}$ \\
\hline M & 74 & $38-41$ & $42-51$ & $10.52-24.39$ & RS & $\mathrm{N}$ & $\mathrm{Y}$ & $\mathrm{Y}$ & $\mathrm{Y}$ & S & $\mathrm{Y}$ & $\mathrm{Y}$ \\
\hline F & 75 & $40-44$ & $45-53$ & $9-20.4$ & RS & $\mathrm{N}$ & $\mathrm{Y}$ & $\mathrm{N}$ & $\mathrm{N}$ & S & $\mathrm{Y}$ & $\mathrm{Y}$ \\
\hline M & 76 & $43-42$ & $51-50$ & $18.6-19.04$ & RS & $\mathrm{Y}$ & $\mathrm{N}$ & $\mathrm{Y}$ & $\mathrm{Y}$ & M & $\mathrm{Y}$ & $\mathrm{N}$ \\
\hline M & 77 & $49-50$ & $54-61$ & $10.2-22$ & RS & $\mathrm{Y}$ & $\mathrm{N}$ & $\mathrm{N}$ & $\mathrm{Y}$ & $\mathrm{O}$ & $\mathrm{Y}$ & $\mathrm{Y}$ \\
\hline F & 78 & $43-47$ & $47-57$ & $8.51-21.27$ & RS & $\mathrm{Y}$ & $\mathrm{Y}$ & $\mathrm{N}$ & $\mathrm{Y}$ & $\mathrm{O}$ & $\mathrm{Y}$ & $\mathrm{Y}$ \\
\hline F & 79 & $39-40$ & $43-48$ & $10.2-20$ & $\mathrm{RS}$ & $\mathrm{N}$ & $\mathrm{Y}$ & $\mathrm{N}$ & $\mathrm{N}$ & $\mathrm{O}$ & $\mathrm{Y}$ & $\mathrm{Y}$ \\
\hline M & 71 & $46-47$ & $50-57$ & $8.7-21.2$ & RS & $\mathrm{N}$ & $\mathrm{Y}$ & $\mathrm{Y}$ & $\mathrm{N}$ & $\mathrm{O}$ & $\mathrm{Y}$ & $\mathrm{Y}$ \\
\hline M & 72 & $54-55$ & $66-67$ & $22.2-21.8$ & RS & $\mathrm{Y}$ & $\mathrm{Y}$ & $\mathrm{Y}$ & $\mathrm{Y}$ & M & $\mathrm{Y}$ & $\mathrm{N}$ \\
\hline M & 75 & $51-56$ & $56-67$ & $9.8-19.29$ & RS & $\mathrm{N}$ & $\mathrm{Y}$ & $\mathrm{N}$ & $\mathrm{Y}$ & $\mathrm{O}$ & $\mathrm{Y}$ & $\mathrm{Y}$ \\
\hline M & 71 & $43-46$ & $54-57$ & $25.58-23.9$ & RS & $\mathrm{Y}$ & $\mathrm{Y}$ & $\mathrm{N}$ & $\mathrm{N}$ & $\mathrm{S}$ & $\mathrm{Y}$ & $\mathrm{N}$ \\
\hline F & 70 & $41-45$ & $50-56$ & $21.9-24.44$ & RS & $\mathrm{Y}$ & $\mathrm{N}$ & $\mathrm{Y}$ & $\mathrm{N}$ & S & $\mathrm{Y}$ & $\mathrm{N}$ \\
\hline M & 74 & $55-57$ & $61-68$ & $10.9-19.2$ & RS & $\mathrm{N}$ & $\mathrm{Y}$ & $\mathrm{N}$ & $\mathrm{Y}$ & $\mathrm{O}$ & $\mathrm{Y}$ & $\mathrm{Y}$ \\
\hline M & 69 & $41-43$ & $47-54$ & $14.6-25.58$ & WRS & $\mathrm{N}$ & $\mathrm{Y}$ & $\mathrm{N}$ & $\mathrm{Y}$ & M & $\mathrm{Y}$ & $\mathrm{Y}$ \\
\hline M & 79 & $43-48$ & $54-60$ & $25.58-25$ & WRS & $\mathrm{Y}$ & $\mathrm{Y}$ & $\mathrm{Y}$ & $\mathrm{N}$ & M & $\mathrm{Y}$ & $\mathrm{N}$ \\
\hline M & 75 & $41-44$ & $46-53$ & $12.1-20.45$ & WRS & $\mathrm{Y}$ & $\mathrm{Y}$ & $\mathrm{N}$ & $\mathrm{N}$ & M & $\mathrm{Y}$ & $\mathrm{Y}$ \\
\hline F & 73 & $45-49$ & $56-60$ & $24.44-22.44$ & WRS & $\mathrm{N}$ & $\mathrm{Y}$ & $\mathrm{Y}$ & $\mathrm{N}$ & M & $\mathrm{Y}$ & $\mathrm{N}$ \\
\hline M & 76 & $48-49$ & $60-62$ & $25-26.5$ & WRS & $\mathrm{Y}$ & $\mathrm{N}$ & $\mathrm{Y}$ & $\mathrm{Y}$ & S & $\mathrm{Y}$ & $\mathrm{N}$ \\
\hline M & 77 & $44-47$ & $50-59$ & $13.63-25.53$ & WRS & $\mathrm{N}$ & $\mathrm{N}$ & $\mathrm{N}$ & $\mathrm{N}$ & S & $\mathrm{Y}$ & $\mathrm{Y}$ \\
\hline M & 78 & $48-53$ & $55-63$ & $10.4-18.86$ & WRS & $\mathrm{N}$ & $\mathrm{Y}$ & $\mathrm{N}$ & $\mathrm{Y}$ & S & $\mathrm{Y}$ & $\mathrm{Y}$ \\
\hline $\mathrm{F}$ & 70 & $51-53$ & $61-65$ & $19.6-22.6$ & WRS & $\mathrm{N}$ & $\mathrm{Y}$ & $\mathrm{Y}$ & $\mathrm{N}$ & S & $\mathrm{Y}$ & $\mathrm{N}$ \\
\hline M & 71 & $41-42$ & $45-51$ & $9.7-17.6$ & WRS & $\mathrm{N}$ & $\mathrm{N}$ & $\mathrm{Y}$ & $\mathrm{N}$ & S & $\mathrm{Y}$ & $\mathrm{Y}$ \\
\hline M & 68 & $49-50$ & $54-61$ & $10.2-22$ & WRS & $\mathrm{Y}$ & $\mathrm{N}$ & $\mathrm{Y}$ & $\mathrm{Y}$ & $\mathrm{O}$ & $\mathrm{Y}$ & $\mathrm{Y}$ \\
\hline F & 72 & $40-44$ & $48-59$ & $20-20.4$ & WRS & $\mathrm{Y}$ & $\mathrm{Y}$ & $\mathrm{Y}$ & $\mathrm{Y}$ & $\mathrm{O}$ & $\mathrm{Y}$ & $\mathrm{N}$ \\
\hline M & 66 & $47-48$ & $54-60$ & $14.89-25$ & WRS & $\mathrm{N}$ & $\mathrm{N}$ & $\mathrm{N}$ & $\mathrm{N}$ & $\mathrm{O}$ & $\mathrm{Y}$ & $\mathrm{Y}$ \\
\hline M & 75 & $41-42$ & $50-50$ & 21.9-19.05 & TIA & $\mathrm{N}$ & $\mathrm{Y}$ & $\mathrm{N}$ & $\mathrm{Y}$ & M & $\mathrm{Y}$ & $\mathrm{N}$ \\
\hline F & 71 & $51-52$ & $56-63$ & $9.8-21.1$ & TIA & $\mathrm{Y}$ & $\mathrm{N}$ & $\mathrm{N}$ & $\mathrm{Y}$ & M & $\mathrm{Y}$ & $\mathrm{Y}$ \\
\hline M & 68 & $54-55$ & $59-65$ & $9.2-18.18$ & TIA & $\mathrm{Y}$ & $\mathrm{Y}$ & $\mathrm{N}$ & $\mathrm{Y}$ & S & $\mathrm{Y}$ & $\mathrm{Y}$ \\
\hline $\mathrm{F}$ & 75 & $39-42$ & $47-51$ & $19.04-17.64$ & TIA & $\mathrm{N}$ & $\mathrm{Y}$ & $\mathrm{N}$ & $\mathrm{N}$ & S & $\mathrm{Y}$ & $\mathrm{N}$ \\
\hline F & 71 & $40-43$ & $49-52$ & $22.5-20.9$ & TIA & $\mathrm{Y}$ & $\mathrm{Y}$ & $\mathrm{Y}$ & $\mathrm{Y}$ & $\mathrm{O}$ & $\mathrm{Y}$ & $\mathrm{N}$ \\
\hline
\end{tabular}

Y - yes, N - no, M - mild, S - severe, O - occlusion, TIA - transient ischemic attack, RS - residual symptoms, WRS - without residual symptoms, MVFCO2 - middle velocity flow, MVFrest - middle velocity flow, CRVC - cerebrovascular reserve capacity, M - male, $\mathrm{F}$ - female. 
The correlation between CVRC in the "affected" carotid artery and the presence of symptoms demonstrate a significant difference at the 0.01 level. Additionally, symptoms and CVRC of the contralateral carotid artery are also significantly correlated at the 0.05 level. Similar results were demonstrated in many relevant studies (Orosz et al. 2002, Ringelstein et al. 1988, Ringelstein et al. 1992, Silvestrini et al. 1996, Telman et al. 2006).

On the contrary, other studies (Nighoghossian et al. 1994, Lucerini et al. 2002) did not find difference in CVRC values between asymptomatic and symptomatic patients. The rationale behind our findings probably lies into the fact that CVRC is also affected in asymptomatic patients, even though they present no symptoms.

The same question arises from a recent study from Schubert et al. (2009) by identifying patients suffering from hemodynamic cerebral insufficiency and could benefit from cerebral revascularization procedures using xenon-CT scanning as a reliable measurement of the critical CRVC. The efficient collateral network forms a deterrent factor in the expression of symptoms.

In a study of asymptomatic patients (Gur et al. 1996), an affected CVRC was found in $47.72 \%$ of patients, whereas other studies showed an affected CRVC in $25 \%$ of patients (combination of SPECT and TCD) (Engelhardt et al. 2004, Barzo et al. 1996, Szabo et al. 1997). In another study by Markus and Cullinane (2001), the affected CVRC was found in the $22.2 \%$ of patients with the $\mathrm{CO}_{2}$ test inhalation but with $8 \%$ concentration. Nevertheless, Orosz et al. (2002) did not confirm affected CVRC in the asymptomatic patients.

We also noticed that the type of the cerebrovascular disease did not affect CVRC values. Specifically, the affected CVRC values in the subgroup of patients with TIA do not significantly differ from the relevant values of the subgroup of patients with symptoms $>24 \mathrm{~h}$ (with or without residual symptoms) $(p=0.056)$. The presence of an infarct and its correlation with the affected CVRC values is also not significant $(p=0.340)$. The results differ from similar studies with the use of PET (Bullock et al. 1985, Naylor et al. 1994). In these studies, the patients with remaining symptoms have affected CVRC in a higher percentage than those without remaining symptoms.

The question that arises whether the affected CVRC is the outcome of brain ischemia due to intracranial artery stenosis or whether extracranial artery stenosis could have deleterious results, too. Naylor et al.
(1994) showed that CVRC returns in its baseline in the $80 \%$ of patients 4 days after CEA and in the rest of the patients 6 months after, demonstrating extracranial artery stenosis as the most significant factor affecting CVRC.

Given the theoretical pedestal of the affected CVRC, an important and reasonable observation is the fact that CVRC follows the degree of the stenosis; the higher the stenosis, the more CVRC is affected. Likewise in NASCET, the risk of infarct reappearance follows the degree of the stenosis. No difference was found between genders (Ferguson et al. 1999).

Nevertheless, other studies conducted in healthy populations (Kerniket et al. 1996, Oláh et al. 2000), showed difference in CRVC between men and women, with women demonstrating higher CVRC values. None of the four risk factors that were examined seems to correlate with the affected CVRC (Kerniket et al. 1996, Oláh et al. 2000). Moreover, because most of the patients usually present with more than one comorbidities, it is not always feasible to establish a predisposing factor without the risk of confounding bias.

It has been clear in asymptomatic or asymptomatic patients, that there is a group of patients with affected CVRC, which reflects in a poor collateral network, a fact that predisposes to ischemia development in asymptomatic patients or stroke recurrence in symptomatic patients. This hypothesis was confirmed by many prospective studies (Kleiser and Widder 1992, Vernieri et al. 1999, Markus and Cullinane 2001, Silvestrini et al. 1996, Gur et al. 1996).

\section{Conclusions}

In conclusion, in this study we demonstrated an association between CVRC and patients with asymptomatic carotid stenosis. These results should be further evaluated and used in caution due to study design. However, it seems that CVRC could be an early markindex to evaluate the risk of stroke in asymptomatic patients and to design their therapeutic approach. Further studies are compulsory in order to justify the use of CVRC in routine clinical practice and to assess the risk of stroke in asymptomatic patients that could eventually benefit from early CEA.

\section{Conflict of Interest}

There is no conflict of interest. 


\section{References}

BARZO P, VÖRÖS E, BODOSI M: Use of transcranial Doppler sonography and acetazolamide test to demonstrate changes in cerebrovascular reserve capacity following carotid endarterectomy. Eur J Vasc Endovasc Surg 11: 83-89, 1996.

BULLOCK R, MENDELOW AD, BONE I, PATTERSON J, MACLEOD WN, ALLARDICE G: Cerebral blood flow and $\mathrm{CO}_{2}$ responsiveness as an indicator of collateral reserved capacity in patients with carotid arterial disease. Br J Surg 72: 348-351, 1985.

DERDEYN CP, GRUBB RL, POWERS WJ: Cerebral hemodynamic impairment. Methods of measurement and association with stroke risk. Neurology 53: 251-259, 1999.

DONAHUE J, SUMER S, WINTERMARK M: Assessment of collateral flow in patients with cerebrovascular disorders. J Neuroradiol 41: 234-242, 2014.

ECST trial: Randomized trial of endarterectomy for recently symptomatic carotid stenosis: final results of the MRC European Carotid Surgery Trial. Lancet 351: 1379-1387, 1998.

ENGELHARDT M, PFADENHAUER K, ZENTNER J, GRIMMER S, WACHENFELD-WAHL C, HEIDENREICH P, LOEPRECHT H, WÖLFLE KD: Impaired cerebral autoregulation in asymptomatic patients with carotid artery stenosis: comparison of acetazolamide-SPECT and transcranial $\mathrm{CO}_{2}$-dopplersonography (in German). Zentralbl Chir 129: 178-182, 2004.

FERGUSON GG, ELIASZIW M, BARR HW, CLAGETT GP, BARNES RW, WALLACE MC, TAYLOR DW, HAYNES RB, FINAN JW, HACHINSKI VC, BARNETT HJ: The North American Symptomatic Carotid Endarterectomy Trial: surgical results in 1415 patients. Stroke 30: 1751-1758, 1999.

GUR AY, BOVA I, BORNSTEIN NM: Is impaired cerebral vasomotor reactivity a predictive factor of stroke in asymptomatic patients? Stroke 27: 2188-2190, 1996.

KARNIK R, VALENTIN A, WINKLER WB, KHAFFAF N, DONATH P, SLANY S: Sex related differences in acetazolamide-induced cerebral vasomotor reactivity. Stroke 27: 56-58, 1996.

KLEISER B, WIDDER B: Course of carotid artery occlusions with impaired cerebrovascular reactivity. Stroke 23: 171-174, 1992.

LUCERTINI G, ERMIRIO D, BELARVI P: Cerebral hemodynamics aspects of severe carotid stenosis: asymptomatic vs symptomatic. Eur J Endovasc Surg 24: 59-62, 2002.

MARKUS H, CULLINANE M: Severely impaired cerebrovascular reactivity predicts stroke and TIA risk with carotid artery stenosis and occlusion. Brain 124: 457-467, 2001.

NAYLOR AR, MERRICK MV, SANDERCOCK PA, GILLESPIE I, ALLEN P, GRIFFIN TM, RUCKLEY CV: Serial imaging of the carotid bifurcation and cerebrovascular reserve after carotid endarterectomy. Br J Surg 80: 1278-1282, 1993.

NAYLOR AR, MERRICK MV, GILLESPIE I, SANDERCOCK PA, WARLOW CP, CULL RE, GRIFFIN TM, RUCKLEY CV: Prevalence of impaired cerebrovascular reserve in patients with symptomatic carotid disease. Br J Surg 81: 45-48, 1994.

NEMOTO EM, YONAS H, KUWABARA H, PINDZOLA RR, SASHIN D, MELTZER CC, PRICE JC, CHANG Y, JOHNSON DW: Identification of hemodynamic compromise by cerebrovascular reserve and oxygen extraction fraction in occlusive vascular disease. J Cereb Blood Flow Metab 24: 1081-1089, 2004.

NIGHOGHOSSIAN N, TROUILLAS P, PHILLIPPON B, ITTI R, ADELEINE P: Cerebral blood flow reserve assessment in symptomatic versus asymptomatic high-grade internal carotid stenosis. Stroke 25: 1010-1013, 1994.

OLÁH L, VALIKOVICS A, BERECZKI D, FÜLESDI B, MUNKÁCSY C, CSIBA L: Gender-related differences in acetazolamide-induced cerebral vasodilatory response: a transcranial Doppler study. J Neuroimaging 10: 151-156, 2000.

OROSZ L, FÜLESDI B, HOKSBERGEN A, SETTAKIS G, KOLLÁR J, LIMBURG M, CSÉCSEI G: Assessment of cerebrovascular reserved capacity in asymptomatic and symptomatic hemodynamically significant carotid stenosis and occlusions. Surg Neurol 57: 333-339, 2002. 
RINGELSTEIN EB, SIEVERS C, ECKER S, SCHNEIDER PA, OTIS SM: Noninvasive and assessment of $\mathrm{CO}_{2}$-induced cerebral vasomotor response in normal individuals and patients with internal carotid artery occlusions. Stroke 19: 963-969, 1988.

RINGELSTEIN EB, VAN EYCK S, MERTENS I: Evaluation of cerebral vasomotor reactivity by various vasodilating stimuli: comparison of $\mathrm{CO}_{2}$ to acetazolamide. J Cereb Blood Flow Metab 12: 162-168, 1992.

SCHUBERT GA, WEINMANN C, SEIZ M, GERIGK L, WEISS C, HORN P, THOMÉ C: Cerebrovascular insufficiency as the criterion for revascularization procedures in selected patients: a correlation study of xenon contrast-enhanced CT and PWI. Neurosurg Rev 32: 29-35, 2009.

SHAIKH NA, BHATTY S, IRFAN M, KHATRI G, VASWANI AS, JAKHRANI N: Frequency, characteristics and risk factors of carotid artery stenosis in ischemic stroke patients at Civil Hospital Karachi. J Pak Med Assoc 60: $8-12,2010$.

SILVESTRINI M, TROISI E, MATTEIS M, CUPINI LM, CALTAGIRONE C: Transcranial Doppler assessment of cerebrovascular reactivity in symptomatic and asymptomatic severe carotid stenosis. Stroke 27: 1970-1973, 1996.

SZABO S, SHETH RN, NOVAK L, ROZSA L, FICZERE A: Cerebrovascular reserve capacity many years after vasospasm due to aneurysmal subarachnoid hemorrhage. A transcranial Doppler study with acetazolamide test. Stroke 28: 2479-2482, 1997.

TELMAN G, KOUPERBERG E, NITECKI S, KARRAM T, SCHWARZ HA, SPRECHER E, HOFFMAN A, YARNITSKY D: Cerebral hemodynamics in symptomatic and asymptomatic patients with severe unilateral carotid stenosis before and after carotid endarterectomy. Eur J Vasc Endovasc Surg 32: 375-378, 2006.

TSIVGOULIS G, ALEXANDROV AV: Cerebral hemodynamics in acute stroke: pathophysiology and clinical implications. J Vasc Interv Neurol 1: 65-69, 2008.

VERNIERI F, PASQUALETTI P, PASSARELLI F, ROSSINI PM, SILVESTRINI M: Outcome of carotid artery occlusion is predicted by cerebrovascular reactivity. Stroke 30: 593-598, 1999.

ZACHRISSON H, FOULADIUN M, BLOMSTRAND C, HOLM J, VOLKMANN R: Functional assessment of highgrade ICA stenosis with duplex ultrasound and transcranial Doppler. Clin Physiol Funct Imaging 32: 241-246, 2012. 